\title{
Glucan and immunotoxicity
}

\section{Editorial}

For decades, glucan has been shown to help overcome the immunosuppressive effects of numerous factors, including chemotherapy and irradiation. Recent studies suggesting a possible new function for glucan have emerged. Not surprisingly, these studies are based on the well-established fact that glucan strongly stimulates all facets of immune response, from nonspecific response to cellular and humoral responses. From this knowledge came the presumption that glucan might be able to overcome or at least lower some immunosuppression caused by toxic agents.

Some interesting experiments have shown that glucans of different sources have a strong protective ability against different mutagenic agents such as 22-aminoanthracene or methyl methyanesulfonate. In these studies, glucans protected the cells by sheltering their DNA and effectively increasing the repair of the double-strand breaks. These studies were further confirmed by findings showing the efficacy of glucan in reducing the damage caused by other mutagenic agents such as doxorubicin, cisplatin, cyclophosphamide and adriamycin. Some of the effects of glucans are attributed to their ability to trap free radicals which are produced during the biotransformation of these chemotherapeutic drugs.

In addition, glucan supports the mitotic activity of bone marrow cells, ${ }^{1}$ which further helps to overcome the negative biological effects of anti-neoplastic drugs. Additional possible effects of glucan on bone marrow cells might be enhancement of the viability of bone marrow cells (shown as increase in bone marrow CFU-GM colony formation and by direct cytotoxocity tests) leading to protection against doxorubicin-induced toxicity. This is particularly important in clinical practice, since myelotoxic injury to the bone marrow is a severe consequence to either total body irradiation or massive chemotherapeutic treatment. The role of glucan in reduction of toxic effects of irradiation/chemotherapy was often explained by the increase of proliferation of bone marrow stem cells. However, direct evidence of this was either missing or completely overlooked. A recent study focused on deposition of iC3b on bone marrow stroma cells and on the interaction of these cells with hematopoietic progenitor cells. The study showed that stroma-iC3b tethers progenitor cells via inserted I-domain of the complement receptor 3 expressed on progenitor cells. The addition of glucan can cause changes in proliferation of tethered progenitor cells with subsequent activation of leukocyte recovery ${ }^{2}$ leading to either faster recovery or higher survival rate observed by many in models including mice and pigs. This hypothesis was further documented by showing that better survival of irradiated animals after transplantation of hematopoietic progenitor cells is CR3-dependent. These findings represent not only a different view on glucan effecting bone marrow, but also on its new role in activating tissue repair.

In addition to bone marrow recovery, glucan has also been found to possess some protective effects against electromagnetic radiation. A recent study using a model of $2.45 \mathrm{GHz}$ electromagnetic radiation and possible oxidative injury of the skin demonstrated oral administration of glucan before the radiation reversed the increase in MDA levels and decrease in SOD activities caused by radiation. Based on these data, authors concluded that orally-given glucan can offer protection
Volume 6 Issue 5 - 2018

\author{
Vetvicka V,Vetvickova J \\ Department of Pathology, University of Louisville, USA
}

Correspondence: Vaclav Vetvicka, Department of Pathology, University of Louisville, Louisville, KY 40202, USA, Fax: 502852 I I77,Email vaclav.vetticka@louisville.edu

Received: October 24, 2018 | Published: October 25, 2018

against oxidative injury induced by electromagnetic radiation, most probably through its anti-oxidant abilities. ${ }^{3}$ These abilities to trap free radicals including singlet oxygen and hydroxyl radical are well established.

Glucan was also tested in the case of mercury poisoning. Global mercury recycling resulted in a significant presence of mercury salts in the environment, with the fish industry being hit particularly hard. In addition, thimerosal (containing an organic ethylmercury), still represent a substance with dangerous immunosuppressive effects. Other mercury salts also possess immunosuppressive and toxic properties. A more detailed study showed that two week exposure of mice to either thimerosal or mercury acetate in drinking water caused significant suppression of humoral and cellular immune reactions such as antibody response, cytokine secretion, phagocytosis, cell proliferation and natural killer activity. However, a two-week feeding with yeast-derived insoluble glucan resulted in significantly lower immunosuppressive effects of mercury. ${ }^{4}$

The mechanisms by which glucan administration blocks or at least lowers mercury-mediated immunosuppression are currently unclear. In addition to plain stimulation of immune reactions, which supplements lower immunity caused by mercury exposure, one can speculate about the activation of some immunologically important genes or about limitation of inflammation and oxidative stress.

Another study focused on glucan's ability to reverse the immunosuppression caused by immunotoxic agents. These experiments used perfluorinated compounds, namely perfluooctane sulphonic acid and perluorooctanic acid, which are bioaccumulative chemicals present in thousands of products. With their known long lifetime in the environment, it is not surprising that these products were detected in the blood of both animals and humans. A human study later showed a decrease in humoral immunity as a result of immunization was caused by heavy exposure to these compounds. Various animal models showed suppression of natural killer cell activity and cell proliferation, suppression of humoral immunity and inhibition of T and IL- 6 and IL-10 secretion.

Our laboratory sought information to determine if glucan can help to reduce the immunotoxic effects of these compounds. Our studies showed that oral exposure to perfluooctane sulphonic acid or perluorooctanic acid resulted in severe immunosuppression, tested by natural killer cell activity, phagocytosis, cell proliferation and antibody response. Most of this suppression was, however, reduced by an oral administration of the glucan. 
These data represent the first study demonstrating that significant immunosuppression caused by environmental pollution can be reduced or partially restored by oral administration of a combination of glucan with other bioactive molecules which might open a new, clinically important pathway of blocking the immunosuppressive or immunotoxic effects of various compounds. In addition, these data could support the possible use of glucan combination in the prophylactic treatment of toxicity caused by perfluorinated compounds and possible additional toxins; however, additional studies must be performed to fully explain these effects.

Another aspect of glucan involvement is in the direct regulation of toxicity of contaminations. The most dangerous and persistent contaminants of feed are aflatoxins. Their presence is mostly controlled by chemical strategies such as fungicides and pesticides, but these treatments usually have a significant negative impact on the environment.

Original reports showed that yeast cell walls can be added to contaminated feeds to selectively bind mycotoxins, causing them to pass the animal digestive tract without any toxic effects on animals or their edible parts. Later, it was shown that the responsible molecule is (1-3)- $\beta$-D-glucan, particularly when having (1-6)- $\beta$-D-glucan side chains. Additional studies demonstrated that these glucan can, under normal conditions, adsorb up to $50 \%$ of zearalenone molecules, ${ }^{5}$ most probably via hydrogen bonding between the hydroxyl, lactone, and ketone groups of the zearalenone molecule with the glucan single helix. These findings were followed by investigations of the interaction between glucan and other mytotoxins, particularly aflatoxin B1. These studies confirmed that glucan is again involved in binding to aflatoxin B1. So far, these applications of glucan as feed additive for decontaminations were proven only with insoluble glucans isolated from Saccharomyces cerevisiae.

A different approach was used by ${ }^{6}$ these authors based their research on previous data showing that manno-oligosaccharides can attenuate aflatoxicosis and that glucans can protect DNA against damage caused by oxidative stress. The group studied the mechanisms involved in the aflatoxin-inhibiting effects of Lentinula edodes cultures. From their experiments, it is clear that these cultures play a role as external stimulus affecting the anti-oxidant status in the fungal cells subsequently inhibiting aflatoxin. Glucan present in the cultures activates the transcription factors related to the anti-oxidant response, which not only adsorb the aflatoxins, but even reduces the aflatoxin production. These results are potentially significant, because L. edodes can grow directly on waste materials such as olive mill wastewaters, potentially inhibiting the production of aflatoxins. As glucans from $L$. edodes have similarly low-to-no cellular toxicity, as the rest of glucans, they can be used directly on animal feeds. The possibility that this type of treatment could simultaneously strengthen the immune system of commercially farmed animals would be an additional bonus.
Some interesting experiments have shown that glucans of different origins have an effective protective activity against different mutagenic agents such as methyl methanesulfonate and 22-aminoanthracene. Glucans protected the cells by sheltering their DNA and increasing the repair of the double-strand breaks (for review see). ${ }^{7}$ Additional studies have also proven the efficacy of glucan in reducing the damage caused by other mutagenic agents such as chemotherapeutic drugs cyclophosphamide, adriamycin (better known as doxorubicin), and cisplatin. The protective effect is attributed to the ability of glucan to trap free radicals produced during the biotransformation of these drugs.

These findings, originally focused on the agricultural industry, were later also demonstrated in aquaculture. Several studies showed that Nile tilapia experimentally infected with aflatoxin B1 exhibited a wide range of organ damage and suppression of defense reactions including phagocytosis, serum bactericidal activity and natural immunity. However, the addition of glucan into feed managed to significantly improve the suppressed activities.

\section{Acknowledgements}

Both authors contributed equally.

\section{Conflict of interest}

The authors have no conflict to declare.

\section{References}

1. Patchen ML, MacVittie TJ. Use of glucan to enhance hemopoietic recovery after exposure to cobalt-60 irradiation. Adv Exp Med Biol. 1982;155:267-272.

2. Cramer DE, Allendorf DJ, Baran JT, et al. Glucan enhances complementmediated hematopietic recovery after bone marrow injury. Blood. 2006;107(2):835-840.

3. Ceyhan AM, Akkaya VB, Gulecol SC, et al. Protective effects of $\beta$-glucan against oxidative injury induced by $2.45-\mathrm{GHz}$ electromagnetic radiation in the skin tissue of rats. Arch Dermatol Res. 2012;304(7):521-527.

4. Vetvicka V, Vetvickova J. Glucan-resveratrol-vitaminC combination offers protection against toxic agents. Toxins. 2012;4(11):1301-1308.

5. CAST Risks in plant, animal, and human systems; Council for Agricultural Science and Technology. Ames, IA, 2003.

6. Reverberi M, Fabbri AA, Zjalic S, et al. Antioxidant enzymes stimulation in Aspergillus parasiticus by Lentinula edodes inhibits aflatoxin production. Appl Microbiol Biotechnol. 2015;69(2):207-215.

7. Mantovani MS, Bellini MF, Angeli JPF, et al. $\beta$-Glucans in promoting health: prevention against mutation and cancer. Mut Res. 2008;658(3):154 161. 